Submitted to the Astrophysical Journal

Preprint typeset using $\mathrm{LAT}_{\mathrm{E}} \mathrm{X}$ style emulateapj v. 11/12/01

\title{
MODELING GALAXY-MASS CORRELATIONS IN DISSIPATIONLESS SIMULATIONS
}

\author{
Argyro Tasitsiomi $^{1}$, Andrey V. Kravtsov ${ }^{1}$, Risa H. Wechsler ${ }^{1,2}$, Joel R. Primack ${ }^{3}$
}

Submitted to the ApJ 2004 May 21

\begin{abstract}
We use high-resolution dissipationless simulations of the concordance flat $\Lambda$ CDM model to make predictions for the galaxy-mass correlations and compare them to the recent SDSS weak lensing measurements of Sheldon et al. (2004). The simulations resolve both isolated galaxy-size host halos and satellite halos (subhalos) within them. We use a simple scheme based on the matching of the circular velocity function of halos to the galaxy luminosity function and on using the observed density-color correlation of the SDSS galaxies to assign luminosities and colors to the halos. This allows us to closely match the selection criteria used to define observational samples. The simulations reproduce the observed galaxy-mass correlation function and the observed dependence of its shape and amplitude on luminosity and color, if a reasonable amount of scatter between galaxy luminosity and circular velocity is assumed. We find that the luminosity dependence of the correlation function is primarily determined by the changing relative contribution of central and satellite galaxies at different luminosities. The color dependence of the galaxy-mass correlations reflects the difference in the typical environments of blue and red galaxies. We compare the cross-bias, $b_{x} \equiv b / r$, measured in simulations and observations and find a good agreement at all probed scales. We show that the galaxy-mass correlation coefficient, $r$, is close to unity on scales $\gtrsim 1 h^{-1}$ Mpc. This indicates that the cross bias measured in weak lensing observations should measure the actual bias $b$ of galaxy clustering on these scales. In agreement with previous studies, we find that the aperture mass-to-light ratio is independent of galaxy color in the range of luminosities probed by observational samples. The aperture mass scales approximately linearly with luminosity at $L_{r}>10^{10} h^{-2} \mathrm{~L}_{\odot}$, while at lower luminosities the scaling is shallower: $M_{\Delta \Sigma} \propto L_{r}^{0.5}$. We show that most of the luminous galaxies $\left(M_{r}<-21\right)$ are near the centers of their halos and their galaxy-mass correlation function at $r \lesssim 100 h^{-1} \mathrm{kpc}$ can therefore be interpreted as the average dark matter density profile of these galaxies. Finally, we find that for galaxies in a given narrow luminosity range, there is a broad and possibly non-gaussian distribution of halo virial masses. Therefore, the average relation between mass and luminosity derived from the weak lensing analyses should be interpreted with caution.
\end{abstract}

Subject headings: cosmology: theory — galaxies: formation — galaxies: halos — large-scale structure of universe

\section{INTRODUCTION}

Understanding the processes that shape the clustering of dark matter and galaxies is one of the main goals of observational cosmology. Modern large redshift surveys, such as the Two Degree Field Galaxy Redshift Survey (2dFGRS, Colless et al. 2001) and the Sloan Digital Sky Survey (SDSS, York et al. 2000), allow measurements of galaxy and galaxy-mass correlations and of their dependence on galaxy properties and environment with unprecedented accuracy.

Concurrently, cosmological $N$-body simulations have developed into a powerful tool for calculating the gravitational clustering of collisionless dark matter in hierarchical cosmologies with well-specified initial conditions. The main obstacle in direct comparisons between models and data is understanding the dependence of theoretical predictions on both the relatively straightforward physics of gravitational clustering and the more complex physics of galaxy formation. The former determines the distribution of dark matter, while the latter affects the relationship

\footnotetext{
1 Dept. of Astronomy and Astrophysics, Kavli Institute for Cosmological Physics, The University of Chicago, Chicago, IL 60637; iro@oddjob.uchicago.edu, andrey@oddjob.uchicago.edu, risa@cf cp.uchicago.edu

2 Hubble Fellow, Enrico Fermi Fellow

3 Physics Department, University of California, Santa Cruz, CA 95064 joel@scipp.ucsc.edu
}

between the distribution of galaxies and mass, the "bias". The complexity of processes operating during galaxy formation on a very wide range of scales makes it difficult to include them directly in simulations, although efforts in this direction are ongoing (e.g., Katz et al. 1999; White et al. 2001; Yoshikawa et al. 2001; Pearce et al. 2001; Berlind et al. 2003; Weinberg et al. 2004).

Given the difficulty of large-scale galaxy formation simulations, two simpler approaches have recently been pursued to make theoretical predictions for galaxy properties. In the first, the galaxies are identified with dark matter halos and subhalos in dissipationless cosmological simulations (e.g., Colín et al. 1999; Kravtsov \& Klypin 1999; Neyrinck et al. 2003; Kravtsov et al. 2004a). In the second hybrid approach, collisionless $N$-body simulations are combined with a semi-analytic treatment of galaxy formation (e.g., Kauffmann et al. 1997; Governato et al. 1998; Kauffmann et al. 1999a,b; Kolatt et al. 1999; Benson et al. 2000a,b; Somerville et al. 2001; Wechsler et al. 2001; Berlind et al. 2003).

Galaxy-galaxy lensing is a relatively new but important observational probe of the relation between galaxies and dark matter. It directly measures the galaxy-mass crosscorrelation function around galaxies of different types and environments (see, e.g., Brainerd et al. 1996; Fischer et al. 2000; Hoekstra et al. 2001, 2004; McKay et al. 2001; Sheldon et al. 2001, 2004; Smith et al. 2001; Wilson et al. 
2001).

Several theoretical studies of the galaxy-mass correlations in cosmological simulations have been carried out in the last three years (Guzik \& Seljak 2001, 2002; Yang et al. 2003; Weinberg et al. 2004). Still, there appear to be several discrepancies between the various model results and observations, which are yet to be properly understood. Guzik \& Seljak (2001) used intermediate-resolution dark matter only simulations combined with a semi-analytic model to identify galaxies and make predictions for the galaxy-mass correlation function. They compared these results to the first weak lensing detection from SDSS (Fischer et al. 2000), and found the amplitude of the galaxymass correlation in their model to be systematically higher. They attributed this discrepancy to the differences between the luminosity function used in their semi-analytic prescription and the observed luminosity function. Yang et al. (2003), using the same theoretical model (intermediateresolution dark matter simulations combined with the Kauffmann et al. 1999a semi-analytic galaxy formation prescription), compared to more extensive observational results from McKay et al. (2001). They also found that the galaxy-mass correlation function in the simulations was systematically higher than the observational measurements, by about a factor of two. Consequently, they found mass-to-light ratios about a factor of two higher than those observed. The study of Weinberg et al. (2004) investigated several of the same statistics, based on galaxies identified in SPH simulations. They found that for systems with small baryonic masses, their dark matter-to-baryon ratios agreed with the mass-to-light ratios, derived from weak lensing data, presented by McKay et al. (2001). For large masses, however, the baryonic masses of their simulated galaxies were high by a factor of 1.5-2 compared to what is required for agreement with the SDSS data. In each of these studies, discrepancies with the data may have been due to discrepancies of the model luminosity functions with that observed. Guzik \& Seljak (2002) used a more phenomenological approach, and developed a formalism in the context of the halo model to fit the McKay et al. (2001) data. They extracted information on, e.g., the dependence of the virial mass-to-light ratio on luminosity, the typical mass for galaxies, and the fraction of galaxies in groups and clusters.

The most recent observational study of galaxy-galaxy lensing by the SDSS collaboration (Sheldon et al. 2004, hereafter S04), has significantly improved the accuracy of galaxy correlation measurements, and may shed light on many of the previous uncertainties. The spectroscopic sample of 127,001 lensing galaxies was combined with more than $9 \times 10^{6}$ source galaxies with photometric redshifts, which allowed a detailed study of the relation between mass and light for several luminosity bands, morphological types, and on scales from $20 h^{-1} \mathrm{kpc}$ to $10 h^{-1} \mathrm{Mpc}$ (physical). In addition to verifying the findings of previous studies, S04 identified new features in the data, including a scale-dependent luminosity (cross) bias.

Besides being a general test of the Cold Dark Matter paradigm, comparisons with cosmological simulations can be used to gain a deeper insight into the interpretation of these new observational results. Furthermore, they can shed light on to what extent weak lensing results can be
TABLE 1

SiMULATION PARAMETERS

\begin{tabular}{cccccc}
\hline \hline Name & $\sigma_{8}$ & $\begin{array}{c}L_{\mathrm{box}} \\
h^{-1} \mathrm{Mpc}\end{array}$ & $N_{\mathrm{p}}$ & $\begin{array}{c}m_{\mathrm{p}} \\
h^{-1} \mathrm{M}_{\odot}\end{array}$ & $\begin{array}{c}h_{\text {peak }} \\
h^{-1} \mathrm{kpc}\end{array}$ \\
\hline$\Lambda \mathrm{CDM}_{60 \mathrm{a}}$ & 1.0 & 60 & $256^{3}$ & $1.07 \times 10^{9}$ & 1.9 \\
$\Lambda \mathrm{CDM}_{60 \mathrm{~b}}$ & 0.9 & 60 & $256^{3}$ & $1.07 \times 10^{9}$ & 1.9 \\
$\Lambda \mathrm{CDM}_{60 \mathrm{c}}$ & 0.9 & 60 & $256^{3}$ & $1.07 \times 10^{9}$ & 1.9 \\
$\Lambda \mathrm{CDM}_{80 \mathrm{a}}$ & 0.75 & 80 & $512^{3}$ & $3.16 \times 10^{8}$ & 1.2 \\
$\Lambda \mathrm{CDM}_{80 \mathrm{~b}}$ & 0.9 & 80 & $512^{3}$ & $3.16 \times 10^{8}$ & 1.2 \\
$\Lambda \mathrm{CDM}_{120}$ & 0.9 & 120 & $512^{3}$ & $1.07 \times 10^{9}$ & 1.8 \\
\hline
\end{tabular}

used to estimate halo masses, or to learn about the average dark matter halo density profiles. In this paper we use high-resolution dissipationless simulations of the concordance $\Lambda$ CDM model to study the galaxy-mass correlation function with specific emphasis on comparing to the observational measurements of S04. Results of several recent studies suggest that gravitational dynamics is the dominant mechanism shaping galaxy clustering, at least in the simple case of galaxies selected above a luminosity or mass threshold (Kravtsov \& Klypin 1999; Kravtsov et al. 2004a; Zentner et al. 2004). Kravtsov et al. (2004a), using darkmatter only simulations which resolve galactic mass subhalos, matched galaxies of a given luminosity to a population of halos and subhalos of a given circular velocity and the same number density, and found excellent agreement with the galaxy-galaxy correlation functions measured in the SDSS. This study extends that approach to investigate the galaxy-mass correlations, and makes detailed comparisons to the new Sheldon et al. (2004) results.

The paper is organized as follows. In $\S 2$ and $\S 3$ we describe the simulations and the halo identification algorithm. The halo samples used in our analysis are described in $\S 4$. The main results, including a detailed comparison with the most recent SDSS measurements, are presented in $\S 7$. In $\S 8$ and $\S 9$ we discuss and summarize our results and conclusions.

\section{SIMULATIONS}

For each simulation in this study we assume the concordance flat $\Lambda$ CDM model: $\Omega_{0}=1-\Omega_{\Lambda}=0.3, h=0.7$, where $\Omega_{0}$ and $\Omega_{\Lambda}$ are the present-day matter and vacuum densities, and $h$ is the dimensionless Hubble constant defined as $H_{0} \equiv 100 h \mathrm{~km} \mathrm{~s}^{-1} \mathrm{Mpc}^{-1}$. This model is consistent with recent observational constraints (e.g., Spergel et al. 2003; Tegmark et al. 2004). To study the effects of the power spectrum normalization, the effects of box size, and cosmic variance, we consider a set of simulations listed in Table 1. The simulations are labeled according to their box size.

Three different simulations of $60 h^{-1} \mathrm{Mpc} \approx 85.71 \mathrm{Mpc}$ box with $256^{3} \approx 1.67 \times 10^{7}$ particles are used. One of these three simulations is normalized to $\sigma_{8}=1.0$, where $\sigma_{8}$ is the rms fluctuation in spheres of $8 h^{-1} \mathrm{Mpc}$ comoving radius. This simulation was used previously to study 
the halo clustering and bias by Kravtsov \& Klypin (1999) and Colín et al. (1999); the reader is referred to these papers for further details. The other two $60 h^{-1} \mathrm{Mpc}$ simulations have a lower normalization of $\sigma_{8}=0.9$, and differ only in the realization of the Gaussian initial conditions. The second subset of simulations followed the evolution of $512^{3} \approx 1.34 \times 10^{8}$ particles in a $80 h^{-1} \mathrm{Mpc} \approx 114.29 \mathrm{Mpc}$ box. These simulations have different power spectrum normalizations of $\sigma_{8}=0.75$ and $\sigma_{8}=0.9$, but were started from the same realization of the initial conditions. We also use a simulation of a $120 h^{-1} \mathrm{Mpc}$ box with $512^{3}$ particles and $\sigma_{8}=0.9$.

The simulations were run using the Adaptive Refinement Tree $N$-body code (ART; Kravtsov et al. 1997; Kravtsov 1999). The ART code reaches high force resolution by refining all high-density regions with an automated refinement algorithm. The criterion for refinement is the mass of particles per cell. In the $\Lambda \mathrm{CDM}_{60 a}$ the code starts with zeroth level uniform $512^{3}$ grid and refines an individual cell only if the mass exceeds $n_{\text {th }}=5$ particles, independent of the refinement level. In terms of overdensity, this means that all regions with overdensity higher than $\delta=n_{\text {th }} 2^{3 L} / \bar{n}$, where $\bar{n}$ is the average number density of particles in the cube, are refined to the refinement level $L$. Thus, for the $\Lambda \mathrm{CDM}_{60 \text { a }}$ simulation, $\bar{n}$ is $1 / 8$. The peak formal dynamic range reached by the code in this simulation is 32,768 , which corresponds to a peak formal resolution (the smallest grid cell) of $h_{\text {peak }}=1.83 h^{-1} \mathrm{kpc}$; the actual force resolution is $\approx 2 h_{\text {peak }}=3.7 h^{-1} \mathrm{kpc}$ (see Kravtsov et al. 1997). In the higher-resolution $\Lambda \mathrm{CDM}_{80}$ simulations the initial grid is $256^{3}$ and the refinement criterion is level- and time-dependent. At the early stages of evolution $(a<0.65)$ the thresholds are set to 2,3 , and 4 particle masses for the zeroth, first, and second and higher levels, respectively. At low redshifts, $a>0.65$, the thresholds for these refinement levels are set to 6,5 , and 5 particle masses. The lower thresholds at high redshifts are set to ensure that collapse of small-mass halos is followed with higher resolution. The maximum achieved level of refinement is $L_{\max }=8$, which corresponds to a comoving cell size of $1.22 h^{-1} \mathrm{kpc}$. As a function of redshift the maximum level of refinement is equal to $L_{\max }=6$ for $5<z<7, L_{\max }=7$ for $1<z<5, L_{\max } \geq 8$ for $z<1$. The peak formal resolution is $h_{\text {peak }} \leq 1.2 h^{-1}$ kpc (physical). The refinement criteria for the $\Lambda \mathrm{CDM}_{120}$ simulation are similar to those of the $\Lambda \mathrm{CDM}_{80}$ runs, except that initially the entire volume is resolved with a $1024^{3}$ grid. The parameters of the simulations are summarized in Table 1.

\section{HALO IDENTIFICATION}

\subsection{The algorithm}

A variant of the Bound Density Maxima halo finding algorithm (Klypin et al. 1999) is used to identify halos and the subhalos within them. The details of the algorithm and parameters used in the halo finder can be found in Kravtsov et al. (2004a). The main steps of the algorithm are identification of local density peaks (potential halo centers) and analysis of the density distribution and velocities of the surrounding particles to test whether a given peak corresponds to a gravitationally bound clump. More specifically, we construct density, circular velocity, and velocity dispersion profiles around each center and it- eratively remove unbound particles using the procedure outlined in Klypin et al. (1999). We then construct final profiles using only bound particles and use these profiles to calculate properties of halos, such as the circular velocity profile $V_{\text {circ }}(r)=\sqrt{G M(<r) / r}$ and compute the maximum circular velocity $V_{\max }$. Our completeness limit is approximately 50 particles. This limit corresponds to the mass below which cumulative mass and velocity functions start to flatten significantly (Kravtsov et al. 2004a). In the following analysis, only halos with masses $M>50 m_{\mathrm{p}}$ are considered.

\subsection{Halo Classification: host halos, satellites, and central galaxies}

In this study we distinguish between host halos with centers that do not lie within any larger virialized system and subhalos located within the virial radii of larger systems. Below the terms satellites, subhalos, and substructure are used interchangeably. To classify the halos, we calculate the formal boundary of each object as the radius corresponding to an enclosed overdensity of 180 with respect to the mean density of the universe. Halos whose centers are located within the boundary of a larger mass halo are classified as subhalos or satellites.

The halos that are not classified as satellites are identified as host halos. Note that the center of a host halo is not considered to be a subhalo. Thus, host halos may or may not contain any subhalos with circular velocity above the threshold of a given sample. The host centers, however, are included in clustering statistics because we assume that each host harbors a central galaxy at its center. Therefore, the total sample of galactic halos contains central and satellite galaxies. The former have the positions and maximum circular velocities of their host halos, while the latter have the positions and maximum circular velocities of subhalos.

In the observed universe, the analogy is simple. In a cluster, for example, the brightest central galaxy that typically resides near the center would be associated with the cluster host halo in our terminology. All other galaxies within the virial radius of the cluster would be considered "satellites" associated with subhalos.

\section{THE GALAXY SAMPLES}

In this study, our galaxy sample is created by assigning realistic SDSS luminosities and colors to dark matter halos. To construct mock galaxy catalogs for comparison with observations, one must define selection criteria for particular halo properties to mimic the selection function of the observational sample as closely as possible. Halo mass is often used to define halo catalogs: e.g., a catalog can be constructed by selecting all halos in a given mass range. However, the mass and radius are poorly defined for the satellite halos due to tidal stripping - which alters a halo's mass and physical extent (see Klypin et al. 1999). Therefore, we use the maximum circular velocity, $V_{\max }$, as a proxy for the halo mass. For isolated halos, $V_{\max }$ and the halo's virial mass are directly related. For subhalos, $V_{\max }$ will experience secular decrease but at a relatively slow rate (Kravtsov et al. 2004b).

To mimic the observational selection function, $r$-band luminosities are assigned to the halos as follows. We match 
the cumulative velocity function $n\left(>V_{\max }\right)$ of the halos to the SDSS observed $r$-band cumulative luminosity function (Blanton et al. 2003c). Note that $n\left(>V_{\max }\right)$ includes both isolated host halos and subhalos. We use the $r$-band data since it is in that band that the SDSS spectra were selected, in which the luminosity function is more reliably measured observationally, and on which most SDSS analyses focus. The mean redshift of SDSS galaxies in the main sample is $z=0.1$, and this is also quite close to the weighted mean for the lens galaxies used by S04. We thus start by matching the $M_{r}(z=0.1)$ luminosity function to the halos identified at $z=0.1$, obtaining the average $V_{\max }-M_{r}$ relation by matching $n\left(>V_{\max }\right)$ to $n\left(<M_{\mathrm{r}}\right)$. This was the same method used to assign luminosities to halos in Kravtsov et al. (2004a), in which galaxy clustering properties were reproduced remarkably well. To match what was done with the S04 magnitudes, we $K$-correct the $z=0.1$ magnitudes to $z=0$ (using the code kcorrect v3_2, described in Blanton et al. 2003a).

Note that the magnitudes in S01 have not been corrected for evolution effects, and we use the Blanton et al. (2003c) luminosity function appropriate for $z=0.1$. The brightest galaxies in the S01 sample are typically at a somewhat higher redshift. Therefore, if we had included evolution effects the galaxy-mass correlation of our brightest sample would be lower than is shown below, since the brightest objects would then be too bright to be included in the corresponding S01 sample. However, the effect is quite small in the magnitude range we consider: smaller than the uncertainty due to cosmic variance and luminosity assignment for galaxies. The evolution correction for the brightest galaxies is less than 0.1 mags, and would result in a less than $10 \%$ correction to the $\Delta \Sigma$ amplitude.

Observations indicate that there is substantial scatter in the relation around the mean Tully-Fisher (TF) and Faber-Jackson correlations for spiral and elliptical galaxies. The magnitude of the scatter varies for different galaxy samples, wavebands, slopes of the correlation, the corrections applied, etc. Willick et al. (1997) found that the TF residuals are consistent with a Gaussian distribution with the standard deviation equal to the rms scatter after various corrections to the absolute magnitudes are applied. The rms values for this scatter in magnitude at a given velocity in red and infrared wavelengths range from $\sim 0.2$ to 0.9 magnitudes (e.g., Aaronson \& Mould 1983; Willick et al. 1997; Verheijen 2001).

Motivated by these findings, we introduce scatter in the relation between $V_{\max }$ and $M_{r}$, assuming that at a given $V_{\max }$ there is a Gaussian distribution of possible $M_{r}$ values. The luminosities are assigned to halos using an iterative procedure that preserves the observed luminosity function. We start by deriving the mean $M_{r}-V_{\max }$ relation by matching the cumulative velocity function in the simulation with the observed luminosity function. Given our assumption of a Gaussian scatter in $M_{r}$ with fixed standard deviation $\sigma_{M_{r}}$ or, equivalently, in natural log luminosity with a fixed standard deviation $\sigma_{\ln L}$, the scatter in velocity at fixed luminosity will be given by $\sigma_{\ln V_{\max }}=$ $d \log V_{\max } / d \log L \times \sigma_{\ln L}$, where the $V_{\max }-L$ relation is taken to be locally well approximated by a power law. As we cannot change the observed galaxy luminosities and luminosity function, to assign a luminosity accounting for

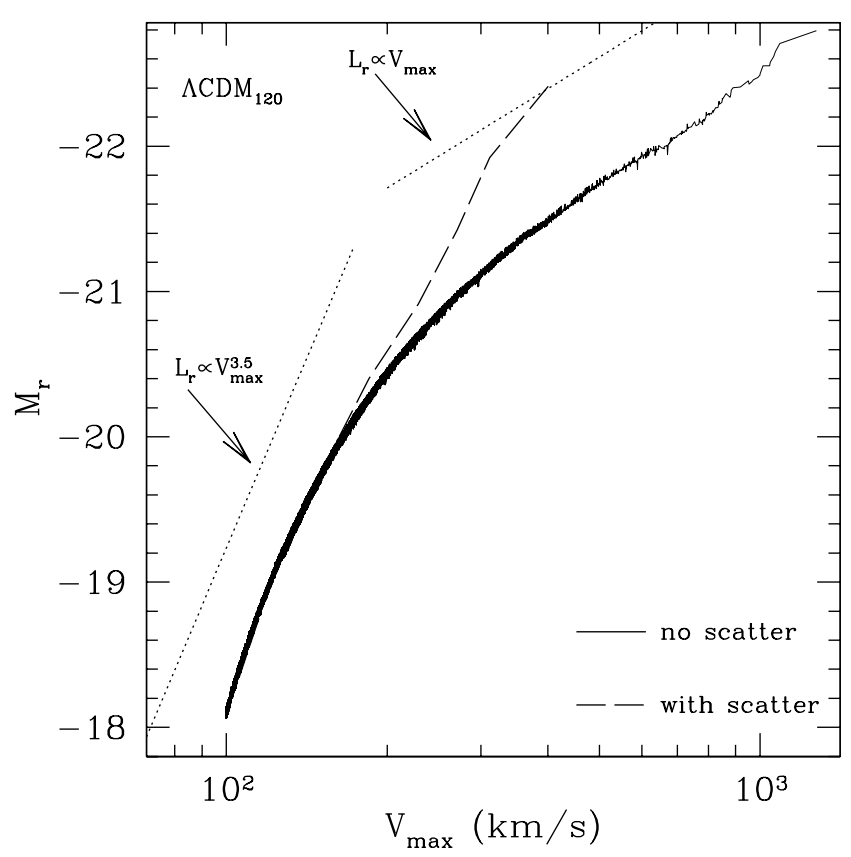

FIG. 1. - The $r$-band absolute magnitude (the magnitudes are $M-5 \log h)$ vs. the maximum circular velocity of halos. The solid curve is obtained by matching the cumulative velocity function $n(>$ $\left.V_{\max }\right)$ to the SDSS luminosity function $n\left(<M_{r}\right)$, both at $z=0.1$. The dashed line denotes the mean $V_{\max }$ as a function of $M_{r}$, after scatter between luminosity and $V_{\max }$ is introduced as described in the text. For comparison, the dotted lines show a power law $L_{r} \propto$ $V_{\max }^{\alpha}$, with $\alpha=3.5$ and $\alpha=1$.

scatter we choose to perturb the values of halo $V_{\max }$ using the expression above and using the mean $V_{\max }-M_{r}$ relation. Note that the maximum circular velocities are perturbed only during the procedure of assigning luminosities to halos and the actual $V_{\max }$ of an object does not change. In each iteration, we thus perturb the velocities by a Gaussian in natural log with this standard deviation, construct a new circular velocity function, and match this to the observed luminosity function to assign new luminosities to halos. The above procedure gives a new mean $M_{r}-V_{\max }$ relation which is used to assign scatter for the next iteration. The iterations are repeated until convergence.

In the analyses presented below we assume a standard deviation of 1.5 magnitudes for the $M_{r}$ distribution at fixed $V_{\max }$. This magnitude of scatter is needed to match the amplitude of the galaxy-mass correlation for the bright samples with the observed values. Although this rms is larger than the values derived from the TF analyses, we note that the latter are derived for the luminosities corrected for the effects of extinction, inclination, etc. We, on the other hand, are applying scatter to the raw luminosities uncorrected for any such effects. For example, the correction due to internal extinction alone can be as high as one magnitude (e.g., Verheijen 2001). We make the simplifying assumption that the scatter is just a function of $V_{\max }$, and do not model it as a function of type for a given $V_{\max }$. Including all galaxy types in a sample is also likely to increase the scatter (Kannappan et al. 2002; Courteau et al. 2003). The magnitude of scatter we assume is therefore perfectly consistent with the observa- 
tions. Given that the scatter does affect the amplitude of the galaxy-galaxy and galaxy-mass correlation for bright galaxies, it should not be neglected. However, since the fiducial value we assume is still uncertain, we note where our results may be sensitive to this assumption.

The relation between the $r$-band absolute magnitude and the maximum halo circular velocity obtained with and without accounting for scatter is shown in Figure 1. The scatter has a significant effect. Without the scatter, the relation has a running slope. Including the scatter transforms the relation into almost a power law $L_{r} \propto V_{\max }^{3.5}$. The effect of scatter is similar to the Malmquist bias: because the abundance of galaxies increases with decreasing luminosity, more low luminosity objects scatter into sample at a fixed mass than high luminosity objects. As we show below, the effect of scatter on the amplitude of $\Delta \Sigma$ is strong at high luminosities and is small at $L \lesssim L_{*}$.

The limited size of the simulation box puts an upper limit on the luminosities of galaxies that can be studied reliably, and the completeness limit of our halo catalogs imposes a lower limit on the luminosities of objects that can be considered. Thus, we will not present a comparison of our results to the S04 sample as a whole, since our sample lacks both the faintest of their objects $(-18 \leq$ $\left.M_{r} \leq-17\right)$, and, most importantly, their brightest bin $\left(-24.0 \leq M_{r} \leq-22.2\right)$. To obtain reliable statistics for objects that bright in simulations, a considerably larger box size is needed. Instead, we try to mimic the intermediate luminosity samples of S04 as closely as possible.

To investigate trends in other bands and with color, magnitudes are assigned to subhalos in the remaining four SDSS bands ( $u, g, i$, and $z$ ) using the observed relation between local galaxy density and color (similar to the procedure described in Wechsler 2004, Wechsler et al. 2004). We first select a volume-limited sample of SDSS galaxies from the first public data release (DR1, Abazajian et al. 2003). We use the CMU-Pitt Value Added Catalog to compile a subsample of these galaxies which is not sensitive to edge effects, and to get a local density measurement for each galaxy: the projected distance to the tenth nearest galaxy neighbor, brighter than $M_{r}-5 \log h=-19.7$ and within $c z=1000 \mathrm{~km} / \mathrm{s}$. We measure the identical quantity for our mock galaxies. For each mock galaxy, we then choose a real SDSS galaxy which has a similar $r$-band luminosity and nearest neighbor distance, and assign the colors of this galaxy to the mock galaxy (bins are chosen to contain $\sim 50$ galaxies). Assigning colors in this way allows us to test whether the color- and band- dependent trends in the data can be reproduced with dark matter halos, under the simple assumption that the dominant variables for determining galaxy colors are its luminosity (or the circular velocity of its subhalo) and the local environment (as is indicated by Blanton et al. 2003b).

\section{ERROR ESTIMATES}

In what follows, all distances, both three-dimensional and projected, are comoving. We study the mass-mass and galaxy-mass two-point correlation functions at distances from $20 h^{-1} \mathrm{kpc}$ up to approximately $15 h^{-1} \mathrm{Mpc}$, except for the galaxy-galaxy correlation function, which cannot be measured down to $20 h^{-1} \mathrm{kpc}$ in our runs. The simulation data is divided into 20 equal logarithmic bins in

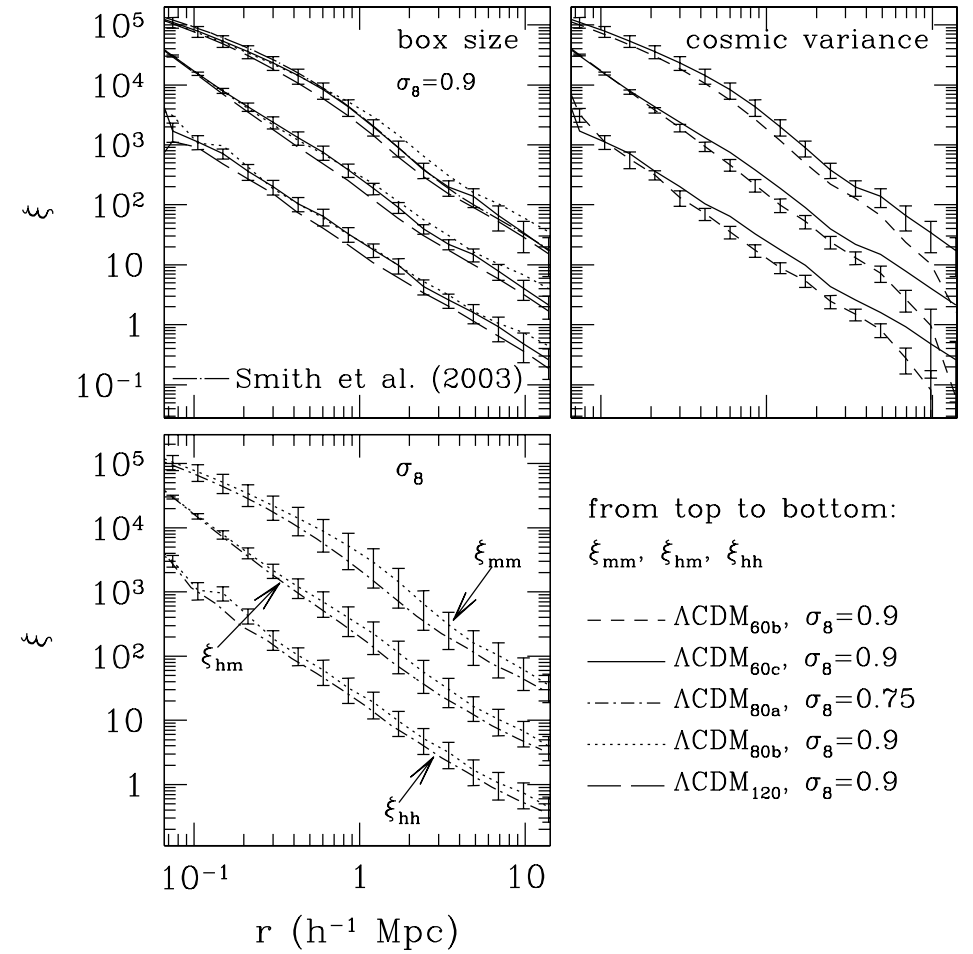

FIG. 2.- Top left panel: The effect of the box size. Top right panel: The effect of cosmic variance. Bottom panel: the effect of power spectrum normalization. In each panel we plot, from top to bottom, the mass-mass $\left(\xi_{\mathrm{mm}}\right)$, the halo-mass $\left(\xi_{\mathrm{hm}}\right)$, and the halo-halo $\left(\xi_{\mathrm{hh}}\right)$ two-point correlation functions. For all boxes, halos (including subhalos) of the same number density are used to calculate the correlation functions. For clarity purposes, the halo-mass and the mass-mass functions are scaled up by a factor of 10 and 100, respectively. The jackknife resampling error bars are plotted. The parameters of the simulations are summarized in Table 1. All correlation functions are presented at $z=0.1$.

$r$. As an estimate of the error in the correlation function in each bin we use the maximum of the Poisson error and the jackknife resampling error. The latter is an estimate of the cosmic variance and is calculated by dividing the simulation box in eight sub-volumes. The jackknife variance $\sigma_{\mathrm{JK}}^{2}$ of the quantity $\chi$ is then

$$
\sigma_{\text {JK }}^{2}=\frac{N-1}{N} \sum_{i=1}^{N}\left(\chi_{i}-\bar{\chi}_{i}\right)^{2} .
$$

Here, $N=8$ is the number of sub-volumes, $\chi_{i}$ is the mean of $\chi$ when the $i$-th sub-volume is excluded, and $\bar{\chi}_{i}$ is the mean of $\chi_{i}$. Typically, the jackknife resampling error is larger than the Poisson error at all scales.

\section{COSMIC VARIANCE AND THE EFFECTS OF BOX SIZE AND $\sigma_{8}$}

In this section, results for the different simulations are compared to understand the effects of the box size, of different power spectrum normalizations $\left(\sigma_{8}\right)$, and of cosmic variance on the two-point correlation functions. The results are shown in Figure 2. In each panel the mass-mass, halo-mass, and halo-halo correlations are plotted from top to bottom, respectively. Each is measured for a volumelimited sample that consists of objects of the same number density for all boxes. The halo-mass and mass-mass cor- 

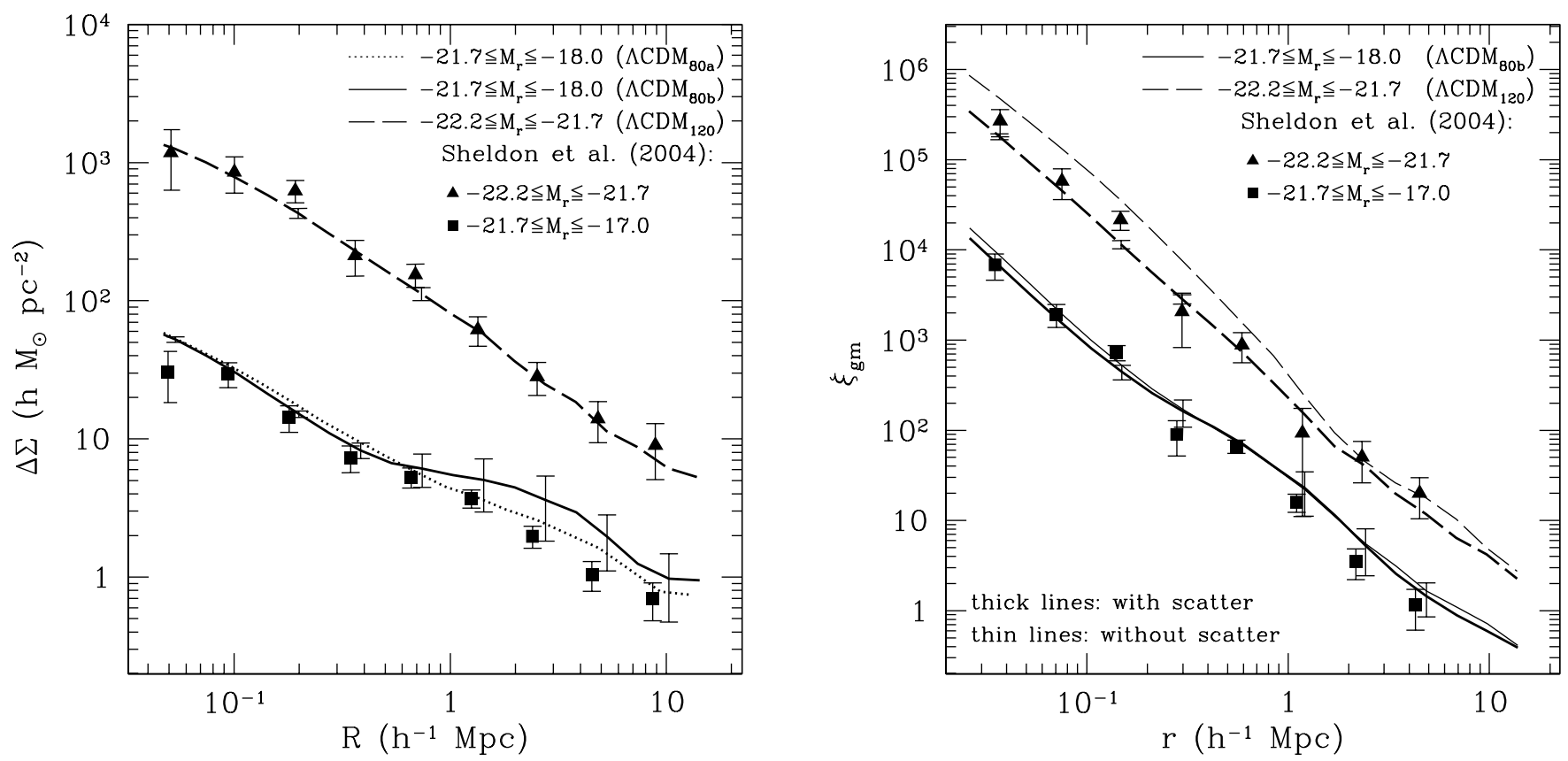

Fig. 3.- Left panel: $\Delta \Sigma$ as a function of projected separation $R$ as measured by Sheldon et al. (2004) (points) and as calculated from the simulations (lines). The results are shown for two $r$-band luminosity bins: $-22.2 \leq M_{r} \leq-21.7$ (triangles/dashed line) and $-21.7 \leq M_{r} \leq-17.0(-18.0)$ (squares and solid line, respectively). The effect of $\sigma_{8}$ can be seen for the fainter sample for which we plot the results for the $\sigma_{8}=0.75$ (dotted line) and $\sigma_{8}=0.9$ (solid line) runs. Right panel: The same as the left panel but showing the (3D) galaxy-mass correlation function. The error bars plotted are $1 \sigma$ jackknife resampling errors. For clarity, error bars in the simulation results are plotted only for scales at which there appears to be some discrepancy between simulations and observations. For illustration purposes, the results for the most luminous bin are shifted upward by a factor of 10 in both panels. Also shown on the right panel with thin lines are the results when no scatter between the luminosity and $V_{\max }$ is introduced. The effect of scatter on $\Delta \Sigma$ is similar.

relation functions are displaced by a factor of 10 and 100 upward, respectively, for clarity.

The effect of simulation box size can be seen in the upper left panel of the figure. Each correlation function is shown here for three different box sizes, all with exactly the same cosmological model and power spectrum normalization. Previous studies based on the mass-mass correlation function have found that the size of the simulation box affects the amplitude on large scales (e.g., Itoh et al. 1992; Colombi et al. 1994; Colín et al. 1999). According to these studies, a small box leads to an underestimate of the correlation function on scales larger than $\sim 10 \%$ of the box size. The finite size of the box sets an upper limit on the longest wavelength of fluctuations that can be present in the simulation. At a given separation, however, the twopoint correlation function, takes (weighted) contributions from all modes. This may be the case with $\Lambda \mathrm{CDM}_{60 c}$ and $\Lambda \mathrm{CDM}_{80 b}$. From our comparison it becomes clear that cosmic variance can significantly alter all these expected trends. Furthermore, while in the linear regime each mode evolves independently, in the nonlinear regime mode coupling occurs and power leaks from large to small scales. We check the effects of non-linearity by using the model developed by Smith et al. (2003) for the calculation of non-linear mass power spectra. The prediction of their model is presented as the long dashed-dotted line hardly discernible in the figure, since the agreement of our simulations with the model is very good.

The upper right panel demonstrates the amount of cosmic variance for the box sizes we use. Simulations $\Lambda \mathrm{CDM}_{60 b}$ and $\Lambda \mathrm{CDM}_{60 c}$ are identical in every respect, except that different random realizations of the initial conditions were used. The comparisons show that the jackknife error may underestimate the cosmic variance somewhat. The effect of varying the value of $\sigma_{8}$ is shown in the lower panel for the two $80 h^{-1} \mathrm{Mpc}$ boxes. Note that these two simulations used the same random realization of the initial conditions. Since the same structures form in both $80 h^{-1}$ Mpc simulations, all differences between the two simulations are thus due to the difference in $\sigma_{8}$.

Keeping in mind these points, in what follows we focus on two of our largest volume simulations, $\Lambda \mathrm{CDM}_{80 b}$ and $\Lambda \mathrm{CDM}_{120}$. The latter provides more robust results at high luminosities $\left(M_{r} \leq-21\right)$ due to better statistics than the smaller volume simulations. Based on the presented box comparisons, the jackknife error bars should be indicative of how different our results can be if another simulation were used.

\section{RESULTS}

\subsection{Weak lensing observables}

We now compare our simulation results to those recently measured by S04 using the SDSS. Using the shapes of the background galaxies, they estimated the tangential shear $\gamma_{\mathrm{t}}$ azimuthally averaged over thin projected radial annuli from the lens galaxy. The average tangential shear is given in terms of the quantities related to the lens mass surface density (Kaiser 1984; Miralda-Escudé 1991). More specifically,

$$
\gamma_{t}=\Delta \Sigma / \Sigma_{\text {crit }}, \quad \Delta \Sigma=\bar{\Sigma}(\leq R)-\bar{\Sigma}(R)
$$

where $\bar{\Sigma}(\leq R)$ is the mean surface density within the projected radius $R, \bar{\Sigma}(R)$ is the azimuthally averaged sur- 
face density at $R$, and $\Sigma_{\text {crit }}$ is the critical density for lensing which depends on the angular diameter distances of the lens and the source. To achieve high signal-tonoise S04 stack the lenses. It is thus desirable to remove the redshift dependence of the signal, which comes in through $\Sigma_{\text {crit }}$. This dependence is removed if one considers $\Delta \Sigma=\gamma_{\mathrm{t}} \times \Sigma_{\text {crit }}$. This is the quantity that S04 measure.

S04 deprojected their $\Delta \Sigma$ to obtain the actual 3D galaxymass correlation function. The deprojection is done via an Abel inversion:

$$
\xi_{\mathrm{gm}}(r)=\frac{1}{\pi \bar{\rho}} \int_{r}^{\infty} \frac{d R}{\sqrt{R^{2}-r^{2}}}\left(\frac{d \Delta \Sigma}{d R}+2 \frac{\Delta \Sigma}{R}\right),
$$

where $r$ is the 3D separation, $R$ the projected separation, and $\bar{\rho}$ the mean density of the universe. S04 assumed for the present-day matter $\Omega_{0}=0.27$ to obtain the mean density $\bar{\rho}$ appearing in the inversion. As discussed, all of our simulations use $\Omega_{0}=0.3$. Thus when comparing to S04 3D results we show their results rescaled by a factor of $0.27 / 0.3$.

We calculate $\Delta \Sigma$ in simulations by selecting objects from the entire halo catalog of a certain simulation in accordance with the magnitude distribution of S04 in a given band (see their Figure 5). The contribution of each object to the correlation function is weighted similarly to the weighting of S04. The weight for each lens depends on $\Sigma_{\text {crit }}$, and thus the redshift of the lens, and on the number of background galaxies available for each lens. For a flux-limited sample, this weight as a function of redshift translates into an effective weight as a function of $r$-band luminosity; to mimic this selection we thus apply the effective weight for the S04 sample to our simulated catalogs, using a function supplied by E. Sheldon.

We first compare our results to the direct observable, $\Delta \Sigma$, which is commonly referred to as the term (projected) correlation function. In the case where the $3 \mathrm{D}$ correlation function is a power law, $\Delta \Sigma$ is the projected correlation function modulo a factor which depends on the slope of the $3 \mathrm{D}$ power law and the average matter density. We also calculate the $3 \mathrm{D}$ galaxy-mass correlation function. In simulations this function can be measured directly rather than through inversion of $\Delta \Sigma$ by using Eq. (3).

\subsubsection{Luminosity dependence}

We compare the projected and the 3D correlation functions, $\Delta \Sigma$ and $\xi_{\mathrm{gm}}$, measured in our simulations to the measurements of S04 in the left and right panels of Figure 3 , respectively. Note that our faintest bin is not exactly the same as that of S04, but the faintest galaxies that are missing from our sample have only a small contribution to the total signal (E. Sheldon, private communication).

In agreement with S04, we find that the amplitude of the correlation function increases with luminosity on intermediate $\left(0.1-1 h^{-1} \mathrm{Mpc}\right)$ scales, while it is nearly independent of luminosity on larger scales. This is indicative of the increase of the effective slope with luminosity. In the low luminosity bin our agreement is better for $\xi_{\mathrm{gm}}$ than for $\Delta \Sigma$. As shown by Eq. (3) the value of the 3D correlation function at given $r$ depends on the behavior of $\Delta \Sigma$ and of its derivative on all scales above $R=r$ (but with contributions closer to $R=r$ more heavily weighted).

As discussed in $\S 4$, we introduce an observationally motivated scatter in the luminosity-maximum velocity relation obtained by matching the luminosity and velocity
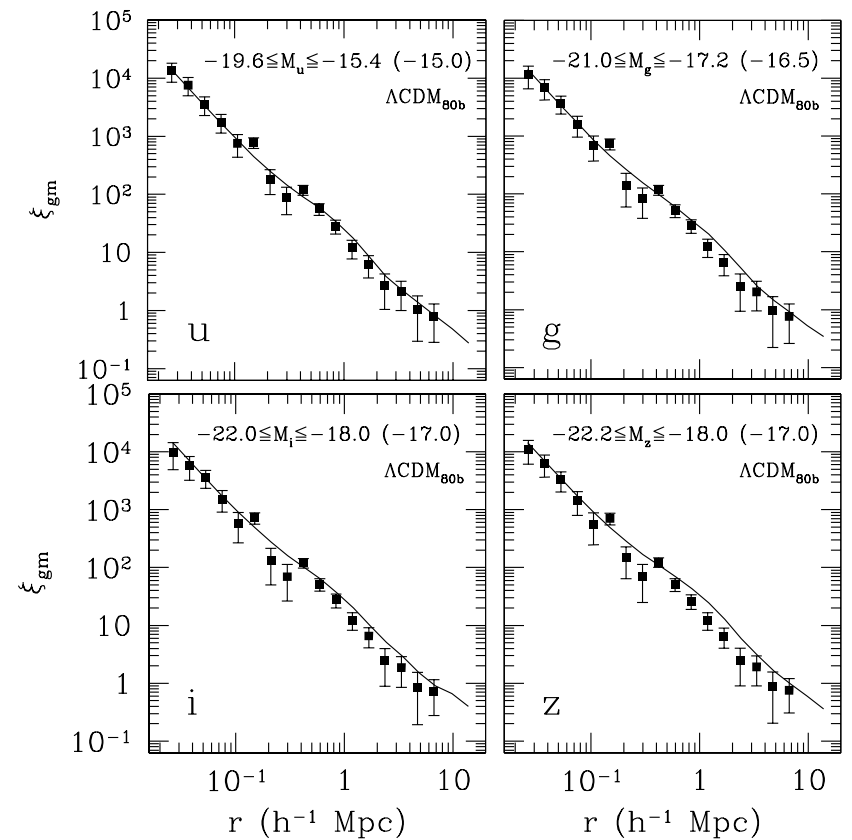

Fig. 4.- Comparison of the simulation galaxy-mass correlation function (lines) with the observations of Sheldon et al. (2004) (points) in the $u, g, i$, and $z$ SDSS bands for the fainter sample. The numbers in parentheses denote the actual faint magnitude limit of the Sheldon et al. (2004) sample in the cases where it does not coincide with the faintest magnitude for which objects are resolved in the simulations.

functions. In the right panel of Figure 3 we plot the results obtained when no scatter between luminosity and velocity is used (thin lines). The net effect of the scatter is to reduce the amplitude of the correlation function by a factor of $\sim 2-3$ in the high luminosity bin, whereas the results for the low luminosity bin are essentially the same, either with or without scatter. This is expected given the flatness of the luminosity function at low luminosities, and its steep fall at high luminosities. The behavior for $\Delta \Sigma$ is similar. In what follows, only results obtained with scatter are shown.

The left panel of Figure 3 also shows the difference in $\Delta \Sigma(R)$ for two simulations with different values of $\sigma_{8}$. Although in $\S 6$ we showed that this effect is smaller than the cosmic variance for boxes of this volume, the two simulations compared here can estimate this effect accurately as they used the same random realization of the initial conditions and thus are not subject to differences due to cosmic variance.

We repeated the analysis in the $r$-band for the other SDSS bands and show the corresponding $\xi_{\mathrm{gm}}$ comparisons for the $u, g, i$ and $z$ bands in Figure 4 for the faintest S04 sample. The luminosities in other bands were assigned using the observed density-color correlation for the SDSS galaxies (see $\S 4$ ). The figure shows that the overall agreement of our simple luminosity scheme, even in the bluer bandpasses, is surprisingly good. The agreement for the intermediate luminosity bin of each band is similar.

\subsubsection{Dependence on color}

As described in $\S 4$, we have assigned colors to our halos based on observational correlation of color and galaxy density. In Figure 5, we compare the correlation functions of 


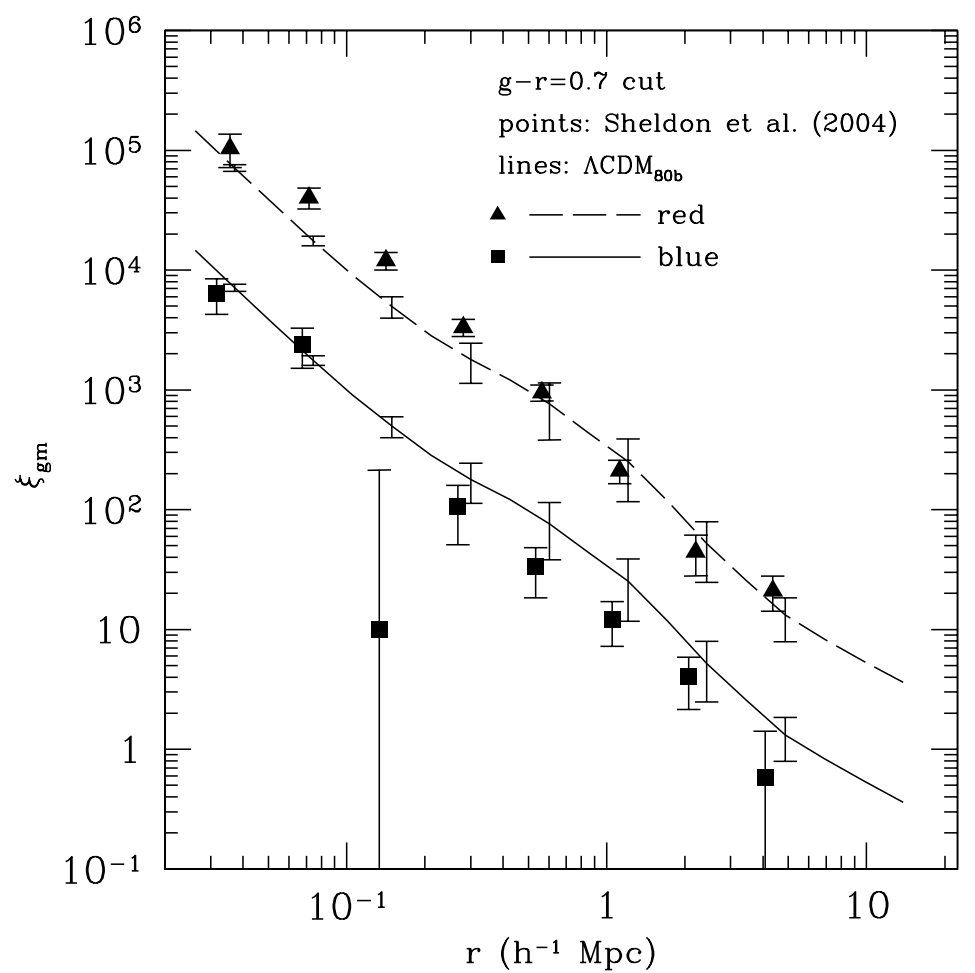

FIG. 5.- The simulation galaxy-mass correlation function for red $(g-r>0.7$, dashed line $)$ and blue $(g-r<0.7$, solid line $)$ objects. The corresponding observational results of Sheldon et al. (2004) are also shown by triangles and squares. For clarity, the results for the red objects are displaced upward by a factor of 10 .

red and blue galaxies separately to those measured by S04. In each case, the color separation is made at $g-r=0.7$, which produces two subsamples with roughly comparable numbers of objects (as does the same selection in the data). The agreement with the data is quite good in both subsamples, especially if we take into account the fact that the simulated sample is missing both the dimmest and the most luminous objects of the S04 total sample, and that cosmic variance is still an effect given the volumes probed here (note that we do not plot results from the larger box here because it is not as complete at the low luminosity end as the simulation shown, but the agreement with the larger box is even better). The agreement indicates that the simple galaxy density-based color assignment in our simulations is sufficient to explain the galaxy-mass correlations in the data.

\subsubsection{The $M_{\Delta \Sigma}-L$ scaling}

To quantify the $\Delta \Sigma$-luminosity scaling, many observational studies use the "aperture mass", $M_{\Delta \Sigma}$ (Fischer et al. 2000; McKay et al. 2001; Smith et al. 2001). This is defined as the quantity obtained by two-dimensional integration of the $\Delta \Sigma(R)$ up to a certain $R$. Following previous studies, we adopt the outer scale of $R=260 h^{-1} \mathrm{kpc}$ (physical). In observational studies this scale has been chosen because it is small enough to avoid a strong contribution from the local environment of the lenses and large enough to measure a significant signal.

The aperture mass simulation results derived in the $r$ band are shown in Figure 6. The data is divided into five

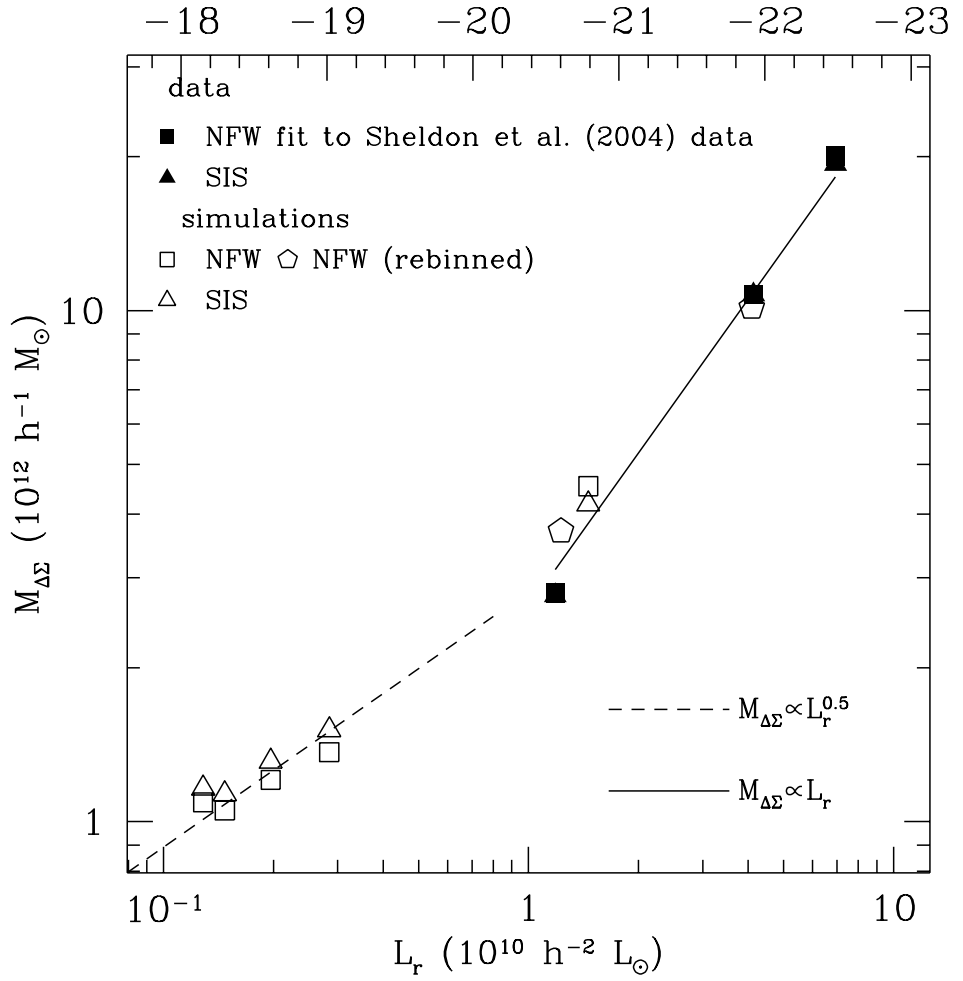

FIG. 6.- The "aperture mass", $M_{\Delta \Sigma}$, as a function of $r$-band luminosity. Solid squares and triangles correspond to the $M_{\Delta \Sigma}$ obtained by fitting the Sheldon et al. (2004) data with a NFW and a SIS profile, respectively. Open squares and triangles correspond to results obtained from simulation when fitting these profiles. For a more direct comparison to the results obtained from the S04 data, the pentagons show the simulation results re-binned to match closely the S04 luminosity bins. All results are derived by using simulation $\Lambda \mathrm{CDM}_{80 \mathrm{~b}}$, with the exception of the highest luminosity bin for which the $\Lambda \mathrm{CDM}_{120}$ run is used to improve the statistics.

bins with comparable number of objects each. For each luminosity bin, we fit the Singular Isothermal Sphere (SIS) and the Navarro, Frenk and White (Navarro et al. 1996, 1997, hereafter NFW) profiles to the $\Delta \Sigma(R)$ in simulations and S04 observations. The selection and weighting of our objects is the same as in S04, but the binning differs: S04 binned their galaxies based on the signal-to-noise ratio, whereas our bins are chosen to have an equal number of objects per bin. For a more direct comparison we also rebin our objects to match the two lower luminosity bins of S04 (pentagons).

In all cases the NFW and SIS fits give comparable $M_{\Delta \Sigma}$. For the SIS fit, $\Delta \Sigma(R)=\Sigma(R)$ (up to an arbitrary constant), but this is not true for the NFW fit. Note that $M_{\Delta \Sigma}$ here refers to the integral of $\Delta \Sigma$ for both fits. For the SIS profile, $M_{\Delta \Sigma}$ is the true projected mass (minus a mass sheet), whereas the interpretation for the NFW profile in terms of an actual mass is more complicated, and one should perceive $M_{\Delta \Sigma}$ simply as the normalization of $\Delta \Sigma$ rather than a true projected mass, a point emphasized by Guzik \& Seljak (2001). A simple interpretation of $M_{\Delta \Sigma}$ as projected mass becomes even more misleading if we take into account that for many galaxy-size halos $260 h^{-1} \mathrm{kpc}$ is larger than the virial radius.

Figure 6 shows a good overall agreement in the $M_{\Delta \Sigma}-$ $-L_{r}$ relation with observations. Differences between the aperture masses of simulations and observations are tracked 


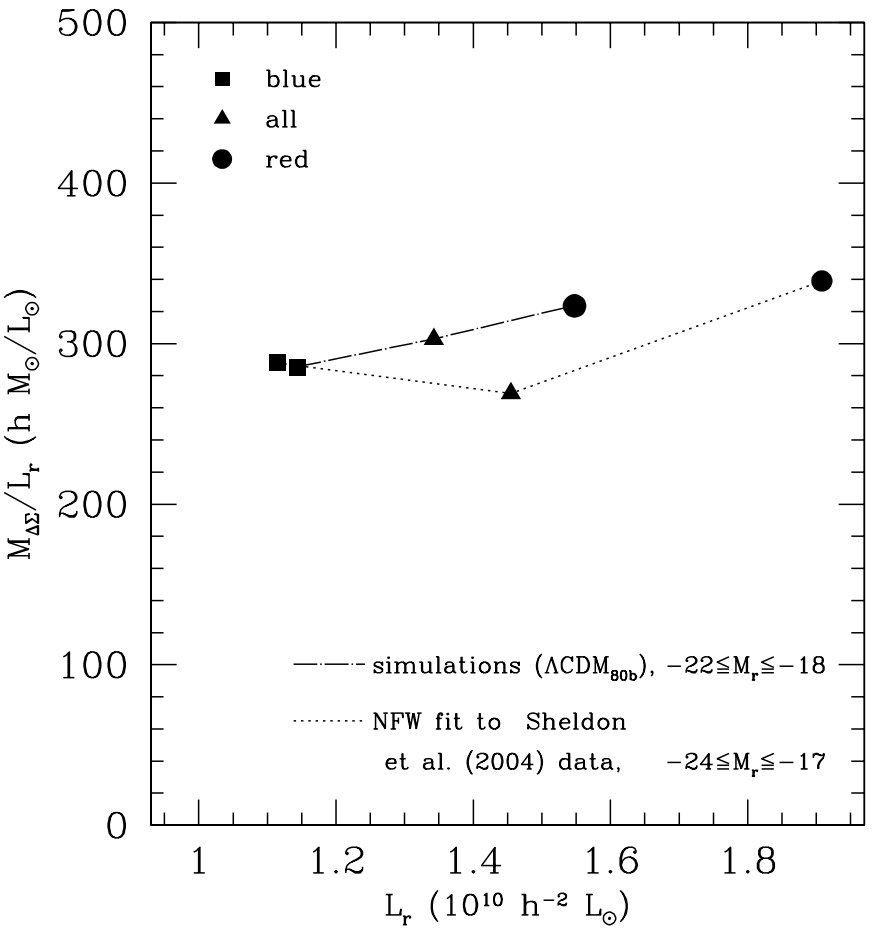

FIG. 7.- The "aperture mass"-to-light ratio, $M_{\Delta \Sigma} / L_{r}$, for the entire sample (triangles), for blue (squares) and red (circles) galaxies vs average luminosity of each sample. Simulation results are connected by the dashed-dotted line, while the values obtained by fitting the $\Delta \Sigma$ measured by Sheldon et al. (2004) with the NFW profile are connected by the dotted line. Slightly different sample definitions are responsible for offsets in the x-axis.

back to the bins not being exactly the same in the two cases. More specifically, the $M_{\Delta \Sigma}$ measured in simulations is somewhat larger than observed for the lowest luminosity bin. This is due to the difference in the faint limits of our and S04 samples: $M_{r}=-18$ and $M_{r}=-17$, respectively: the contribution from galaxies with $-17<M_{r}<-18$ decreases the $M_{\Delta \Sigma}$ and the amplitude of $\Delta \Sigma(R)$ somewhat, as previously mentioned.

The simulations reproduce the $M_{\Delta \Sigma} \propto L_{r}$ scaling of the aperture mass with luminosity found by McKay et al. (2001) reasonably well. Interestingly, our results suggest that the correlation function flattens significantly for $L_{r} \lesssim$ $10^{10} h^{-2} \mathrm{~L}_{\odot}$. This is in agreement with McKay et al. (2002), who found that most of the variation of mass with luminosity is seen for $L_{r} \gtrsim 1.5 \times 10^{10} h^{-2} L_{\odot}$ galaxies. As is discussed below, such a shallowing is also observed for the host halo mass-to-light ratio (van den Bosch et al. 2003; Vale \& Ostriker 2004).

Using this aperture mass measurement, McKay et al. (2001) calculated mass-to-light ratios for the entire sample and for samples separated by galaxy type, and found that the $M_{\Delta \Sigma} / L$ ratio in the red wavebands is insensitive to morphology. In Figure 7 we compare the $M_{\Delta \Sigma} / L_{r}$ calculated for our sample with that obtained by fitting a NFW profile to the S04 sample (this corresponds to the masses designated by solid squares in Figure 6). In addition to the value for the entire sample, we also show values for the blue and red subsamples defined with the color cut of $g-r=0.7$ (see $\S 4$ and $\S 7.1 .2$ ). The figure shows that the $M_{\Delta \Sigma} / L_{r}$ ratio is fairly constant and thus insensitive to the color of the galaxies. The average luminosities of the red and blue simulation subsamples differ from that of

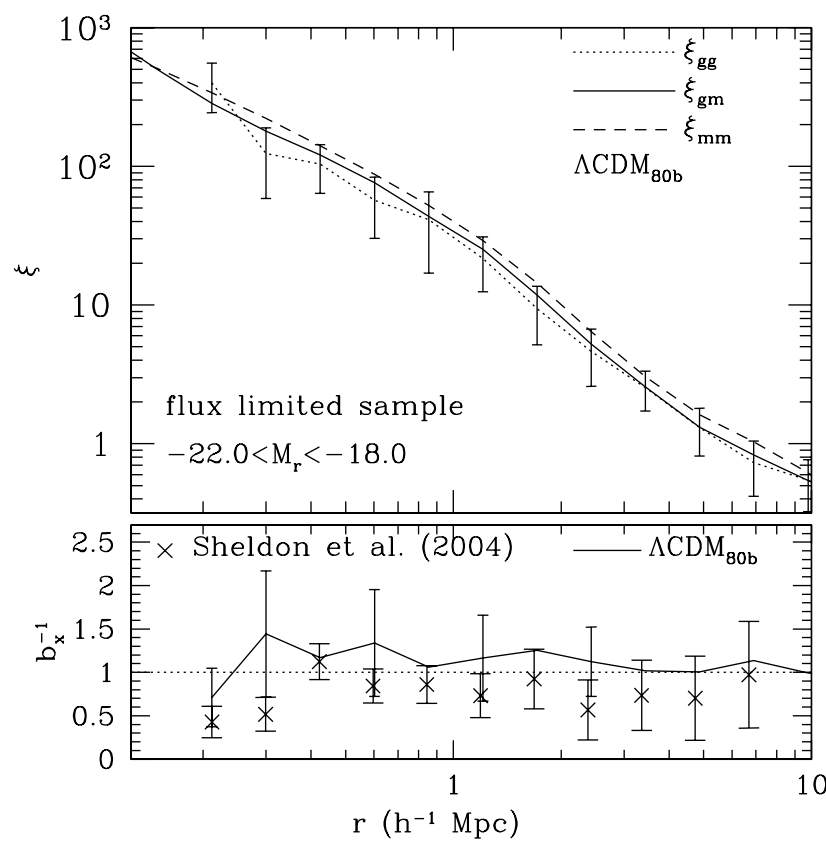

FIG. 8.- Top panel: The galaxy-galaxy, the galaxy-mass and the mass-mass correlation functions. For the galaxy-galaxy and the galaxy-mass correlations galaxies are selected according to the SDSS selection function. Only objects with $-22<M_{r}<-18$ are used, close to the flux limited samples of Sheldon et al. (2004) and Zehavi et al. (2004). Bottom panel: Inverse cross-bias, $b_{x}^{-1}$, as estimated by Sheldon et al. (2004) (points) and as measured in our simulations (solid line). For clarity, error bars in the simulation results are plotted only for scales at which there appears to be some discrepancy between simulations and observations.

the observational subsamples since in simulations we miss both the dimmest and the most luminous objects. Note that for the data of S04 we find approximately $50 \%$ higher values of these ratios than were found by McKay et al. (2001) for their sample. Our results differ from S04 less than the aperture mass-to-light ratio for their blue and red subsamples differ.

\subsection{Bias}

The galaxy bias is a measure of how well galaxies trace the underlying mass distribution. It is often characterized by the bias parameter, $b$, defined in terms of the galaxygalaxy and the mass-mass correlation functions (Kaiser 1984)

$$
b^{2}=\frac{\xi_{\mathrm{gg}}}{\xi_{\mathrm{mm}}} .
$$

The correlation coefficient, $r$, relates the galaxy-mass correlation function to the mass-mass and galaxy-galaxy correlation functions (Pen 1998):

$$
r=\frac{\xi_{\mathrm{gm}}}{\left(\xi_{\mathrm{mm}} \xi_{\mathrm{gg}}\right)^{1 / 2}} .
$$

The mass-mass correlation function is not directly measurable, but weak lensing observations of the galaxy-mass correlations can be combined with measurements of the galaxy-galaxy correlation function to estimate the crossbias parameter,

$$
b_{x}=\frac{\xi_{\mathrm{gg}}}{\xi_{\mathrm{gm}}}=\frac{b}{r} .
$$


Figure 8 compares the inverse cross bias, $b_{x}^{-1}$, measured from simulations with that measured by S04 for a fluxlimited sample. S04 use the inverse galaxy-mass bias because their $\xi_{\text {gm }}$ is much noisier than $\xi_{\text {gg }}$. Their flux- limited sample, used to obtain $\xi_{\mathrm{gm}}$, spans the $r$-band magnitude range $-24<M_{r}<-17$. For $\xi_{\text {gg }}$ they use the results of Zehavi et al. (2004) for a flux-limited sample with $r$ band magnitudes in the range $-22.2<M_{r}<-18.9$ and selection function similar to that of S04. S04 corrected for the difference in average luminosity of the two samples by rescaling the correlation length of Zehavi et al. (2004) using its scaling with luminosity in $2 \mathrm{dF}$ (Norberg et al. 2001). For the simulation results we use objects in the range $-22<M_{r}<-18$ for both $\xi_{\text {gm }}$ and $\xi_{\text {gg }}$ and select objects to mimic the selection function of S04. The figure shows that the cross-bias in simulations is in very good agreement with observations. Note that, again, we are missing the dimmest and the brightest of the objects used by $\mathrm{S} 04$. The cross-bias is approximately scale-independent with $b_{x} \approx 1$.

Figure 9 shows the correlation coefficient $r$ and bias $b$ for two volume-limited simulation samples of different luminosities. In the top panel we also show comparisons of the cross-bias, $b_{x}^{-1}$, for the volume-limited sample of S04. The simulation results agree with observations within the error bars. A small offset of the simulation results toward smaller values of $b_{x}$ may be due to objects with $-23.0<M_{r}<-22.5$, which are not present in our simulations due to the small box size and which could enhance the clustering signal somewhat.

Semi-analytic galaxy formation models suggest that $b_{x}$ is a direct measure of the bias $b$ on large scales, or, equivalently, that the correlation coefficient $r$ approaches unity for $r \gtrsim 1 h^{-1}$ Mpc (Guzik \& Seljak 2001; Berlind \& Weinberg 2002). Figure 9 shows that the correlation coefficient is indeed approximately unity on scales $\geq 1 h^{-1} \mathrm{Mpc}$ in our simulations. This therefore confirms that on these scales the cross bias is expected to be a fair measure of the standard bias $b$ for both bright and faint samples.

The scale dependence of bias measured here is in agreement with previous studies (Colín et al. 1999). Note that the lower luminosity sample exhibits an "antibias" at $r \lesssim$ $3 h^{-1} \mathrm{Mpc}$. For the high-luminosity sample the shape of the bias profile is similar to that of the low-luminosity sample: it is scale-independent on large scales and decreases on smaller scales. The decrease on small scales has been attributed to dynamical friction and tidal destruction processes in the high density regions of clusters and groups (Kravtsov \& Klypin 1999; Zentner et al. 2004). An increase at small separations, $r \lesssim 0.5 h^{-1} \mathrm{Mpc}$, is likely due to the tendency of the brightest galaxies to lie near the center of groups and clusters (Seljak 2000; Weinberg et al. 2004). For the same reason the correlation coefficient of the bright subsample increases above unity for separations $r \lesssim 1 h^{-1} \mathrm{Mpc}$.

\section{DISCUSSION}

\subsection{Luminosity dependence of galaxy-mass correlations}

In the previous section we showed that simulations can reproduce the luminosity dependence of the galaxy-mass correlation function observed for the S04 sample, if a reasonable amount of scatter between $V_{\max }$ and luminosity is
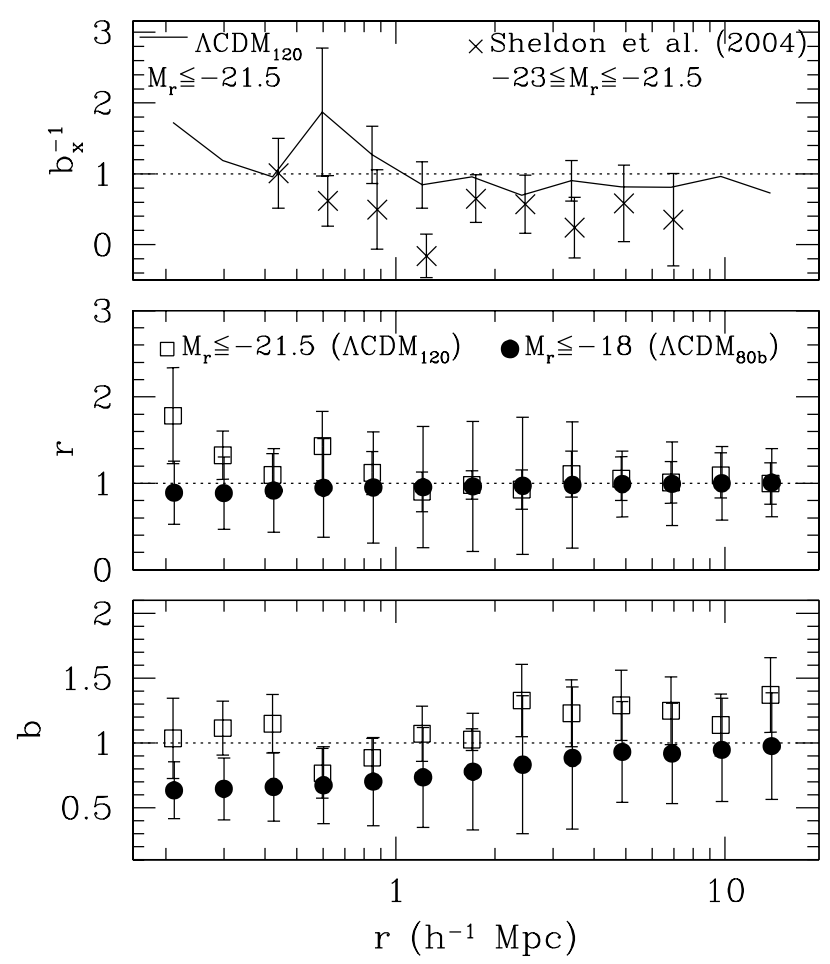

FIG. 9.- Bias for the volume-limited samples. Top panel: Inverse cross-bias, $b_{x}^{-1}$, as estimated by Sheldon et al. (2004) (crosses) for a sample with $-23 \leq M_{r} \leq-21.5$ and as measured in our simulations (solid line) for objects with $M_{r} \leq-21.5$. For clarity, error bars in the simulation results are plotted only for scales at which there appears to be some discrepancy between simulations and observations. Middle panel: Correlation coefficient as a function of scale for two volume-limited simulation samples with $M_{r} \leq-18$ (solid circles) and $M_{r} \leq-21.5$ (open squares). Bottom panel: Bias as a function of scale for the same samples as in the middle panel.

assumed. The luminosity dependence of these correlations is weak at large scales $\left(\gtrsim 1 h^{-1} \mathrm{Mpc}\right)$ and strong at small scales $\left(0.1<R<1 h^{-1} \mathrm{Mpc}\right)$. To understand the origin of this behavior, we compute contributions to the correlation function $\xi_{\mathrm{hm}}$ from host halos ("central galaxies") and from halos classified as satellites (see $\S 3$ ). Figure 10 shows the results for the simplest case of the volume-limited samples with $r$-band luminosities in four different bins, [-19, -18], $[-20,-19],[-21,-20]$, and $[-22,-21]$. We adopt the convention of including the -1 term appearing in the definition of the total correlation function in the host halo term.

The bottom panels of Figure 10 show that the relative contribution of the host halos compared to that of satellites is considerably larger at high luminosities, as expected. The central galaxy contribution at scales $r \lesssim$ $1 h^{-1}$ Mpc changes with luminosity only in amplitude, not in shape. At the high luminosity end, most galaxies are central galaxies and the increase of the correlation amplitude with luminosity reflects the increase in average mass and density of their host halos (Berlind \& Weinberg 2002). At low luminosities, and at intermediate-large separations, the satellite contribution is significant and leads to the development of a 'shoulder' in the overall correlation function, since outside the typical satellite radius, one starts to measure the density of the environment in which the satellites are embedded. The existence of this feature makes the total correlation function at relatively low luminosities ef- 


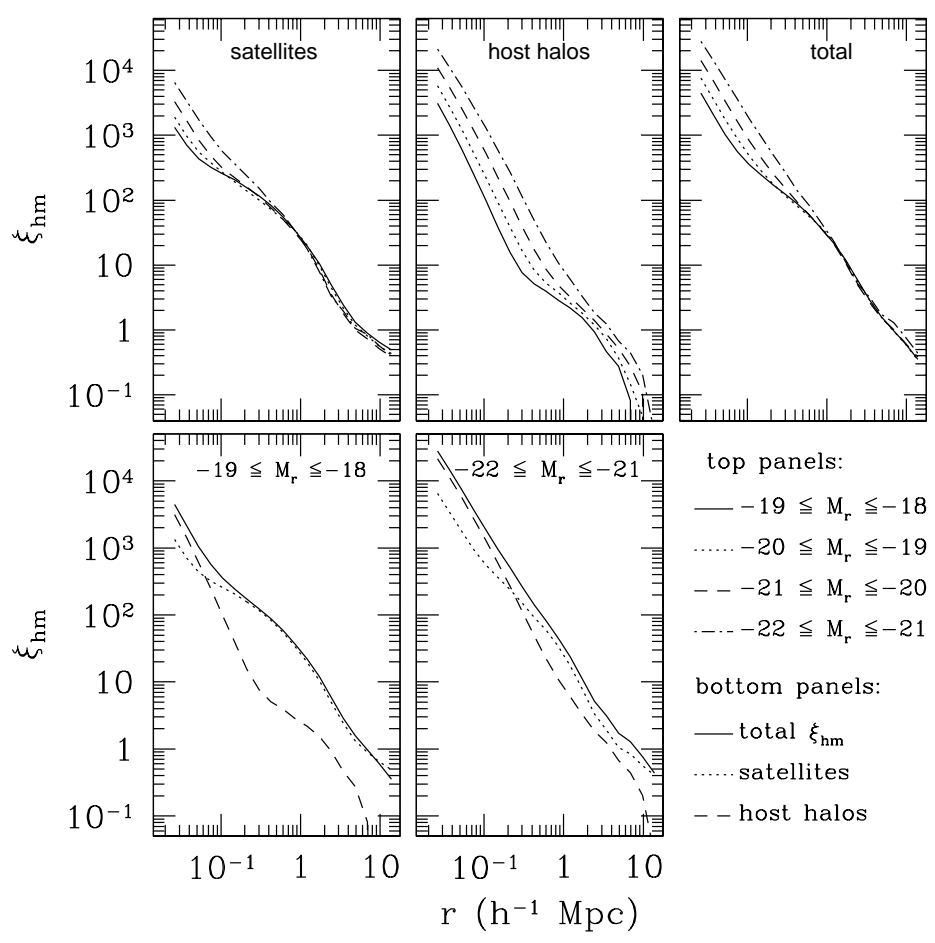

FIG. 10. - Top row of panels: Volume-limited halo-mass correlation function for four different $r$-band luminosity bins and contributions to the correlation function from satellite and host halos. Upper left panel: The satellite contribution. Middle upper panel: The contribution of host halos. Right upper panel: total correlation function. Lower left panel: The (total) halo-mass correlation function (solid line) for the $-19 \geq M_{r} \geq-18$ luminosity bin, the satellite contribution (dotted line), and the host halo contribution (dashed line) for the dimmest luminosity bin. Lower right panel: The same as in the lower left panel but for the brightest luminosity bin.

fectively shallower than that at high luminosities.

\subsection{Average halo masses and density profiles from weak lensing}

An important question about weak lensing observations is whether they will allow us to extract information about the average halo mass and mass profile of the lenses. Stacking the lenses to achieve high signal-to-noise makes the interpretation of the average mass difficult. It has been noted (see, e.g., Guzik \& Seljak 2001; Yang et al. 2003) that complications arise for several reasons including the scatter in sizes and masses of objects and the fact that a fraction of galaxies may be located within a group or cluster of galaxies.

These complications could be limited if only the central galaxy of each halo was used to measure the correlation signal. However, even in this case the interpretation of mass is not straightforward. In Figure 11 we show the distribution of virial masses ${ }^{4}$ for central galaxies in four luminosity bins. The distributions in cases with and without scatter are shown. The distribution of masses in the case without scatter has a finite width because there is scatter between virial mass and $V_{\max }$. The figure shows that introducing scatter between $V_{\max }$ and $L_{r}$ significantly broadens the mass distributions and makes them non-gaussian. The broadening is largest in the most luminous bin. As a re-

${ }^{4}$ Recall that the virial mass is defined as the mass within the radius at which an overdensity of 180 times the mean matter density of the universe is reached.
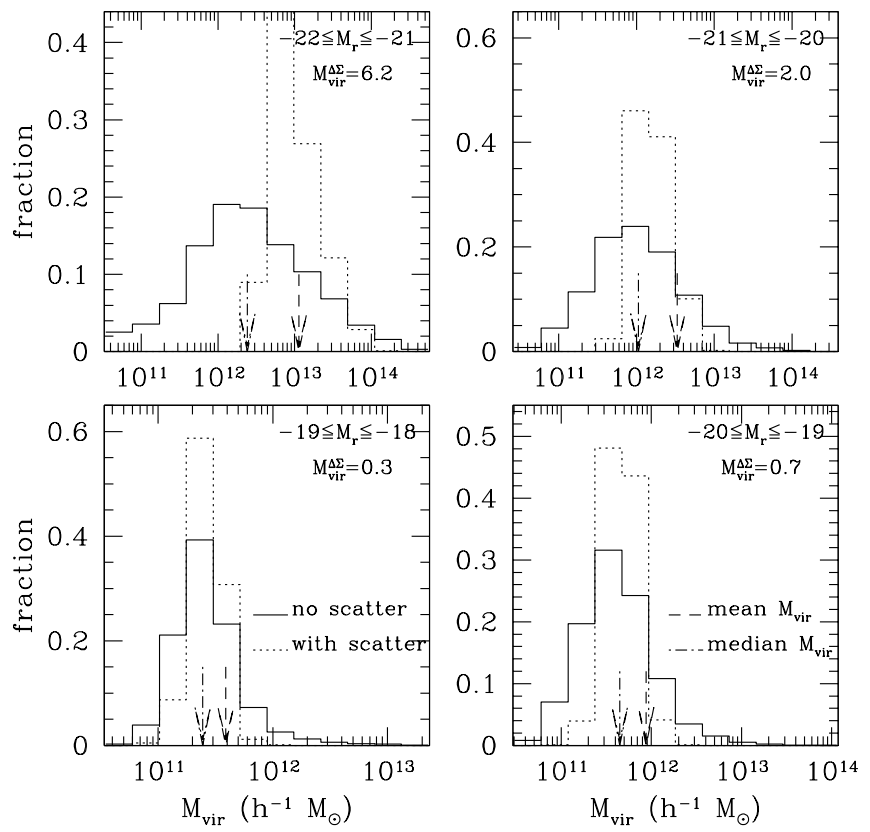

FIG. 11.- Halo virial mass distributions (defined using an overdensity of 180 with respect to the mean density of the universe) for central galaxies in four luminosity bins with and without scatter in the $V_{\text {max }}$-luminosity relation (solid and dotted histograms, respectively). $M_{\text {vir }}^{\Delta \Sigma}$ is the virial mass obtained by fitting the $\Delta \Sigma$ of the corresponding subsample of galaxies, in units of $10^{12} h^{-1} M_{\odot}$. For the distributions with scatter, the mean and median virial masses are indicated with dashed and dot-dashed arrows, respectively.

sult, it becomes difficult to assign a specific mass to a given (even narrow) luminosity range. For example, the figure shows that the mean mass is significantly larger than the median for every luminosity bin. The best fit virial mass, $M_{\text {vir }}^{\Delta \Sigma}$, obtained by fitting $\Delta \Sigma$ for the corresponding galaxy sample to a NFW profile quoted in the figure is a different effective averaging, and gives values between the median and the mean masses.

In agreement with Yang et al. (2003), we find a tight correlation between $M_{\mathrm{vir}}^{\Delta \Sigma}$ and the aperture mass $M_{\Delta \Sigma}$. Both quantities are derived from the same fit, and thus a correlation is expected, but without information on the halo concentrations, one cannot be derived from the other. Nevertheless, as Yang et al. (2003) showed, this correlation is recovered easily if one assumes the well established correlation between the virial mass and concentration (Bullock et al. 2001), which in the absence of scatter between concentration and virial mass renders the NFW effectively a one parameter fit. Given the averaging done to obtain $\Delta \Sigma$, the average concentration-mass correlation is sufficient. In agreement with Yang et al. (2003), we find the following scaling for both central galaxies and galaxies in low density environments

$$
M_{\mathrm{vir}}^{\Delta \Sigma}=(0.31 \pm 0.01) \times M_{\Delta \Sigma}
$$

Here both masses are in units of $10^{12} h^{-1} M_{\odot}$. The scaling is exactly the same when no scatter is used, since this relation reflects the properties of the average mass density profiles of our dark matter halos.

The existence of significant scatter between luminosity and mass even for central galaxies indicates that the mass, 
$M_{\text {vir }}^{\Delta \Sigma}$, derived from weak lensing cannot be interpreted in a straightforward way as the mass for galaxies of a given luminosity. Instead, there is a rather broad distribution of masses. The mass-luminosity relations should therefore be quoted and interpreted with caution. Figure 11 shows that the amplitude of the relation will depend on which mass one considers. The actual distribution depends on the still uncertain details of the scatter between mass and light, but even if the true relation is different from what we have assumed here, it will be incomplete if the distribution of masses is not specified. In conclusion, the mass distribution at fixed luminosity can be wide enough so that the concept of a mean mass becomes obscure. Referring to $M_{\mathrm{vir}}^{\Delta \Sigma}$, the mass of $L_{*}$ galaxies that we find is $\approx 2 \times 10^{12} h^{-1} \mathrm{M}_{\odot}$ as shown for the $-21 \leq M_{r} \leq-20$ bin in Figure 11 and is in general agreement with the mass found by Yang et al. (2003) and the best fit values derived by Guzik \& Seljak (2002) from the fit to the SDSS weak lensing data.

A related question is whether a meaningful average density profile of halos can be recovered from lensing data. Our results show that here, again, one has to focus on either the central galaxies or galaxies in low density environments. As we show in $\S 8.1$, for luminous galaxies the galaxy-mass correlation is dominated by the contribution of central galaxies, especially at small scales. For high-luminosity samples it is therefore meaningful to interpret $\Delta \Sigma(R)$ in terms of the average halo density profile at $r \lesssim 100-200 h^{-1} \mathrm{kpc}$.

\section{CONCLUSIONS}

We have presented a detailed comparison of galaxymass correlation functions measured in high-resolution cosmological simulations of the concordance $\Lambda$ CDM cosmology with the most recent weak lensing SDSS observations. We found that the simple recipe of assigning a luminosity to a halo of a certain maximum circular velocity by matching the subhalo velocity function in simulations to the observed luminosity function leads to good agreement with the observed galaxy-mass correlation and its dependence on luminosity, if an observationally-motivated amount of scatter is introduced in the $V_{\max }-L_{r}$ relation. Our main results and conclusions can be summarized as follows:

- The simulations reproduce the galaxy-mass correlation function measured by Sheldon et al. (2004) in SDSS and the observed dependence of its shape and amplitude on luminosity. Interestingly, the agreement for bright samples seems to require some scatter between luminosity and circular velocity. The amount of scatter required is consistent with the observed scatter.

- The galaxy-mass correlation function for central galaxies is a strong function of the galaxy luminosity (halo velocity), while $\xi_{\text {gm }}$ for satellite galaxies is only weakly sensitive to luminosity. The luminosity dependence of the correlation function as a whole is thus determined primarily by the increasing contribution of bright central galaxies relative to the satellite galaxies at bright luminosities. Conversely, the correlation function gets shallower with decreasing luminosity because it is increasingly dominated by the contribution from the satellite (non-central) galaxies in halos at intermediate and large scales.

- We use the color-density correlation observed in the SDSS to assign colors to the galactic halos in simulations and compare the simulation results to observations in other SDSS bands, from $u$ to $z$. In each band the agreement between galaxy-mass correlation function in simulations and observations is remarkably good. The simulations also reproduce the observed trend of the galaxy-mass correlation function with the $g-r$ color.

- In agreement with previous studies, we find that the aperture mass-to-light ratio, $M_{\Delta \Sigma} / L_{r}$, is independent of galaxy color. For $L_{r} \gtrsim 10^{10} h^{-2} L_{\odot}$, $M_{\Delta \Sigma} / L_{r}$ is approximately independent of luminosity. For both central galaxies and galaxies in low density environments the best fit virial mass $M_{\text {vir }}^{\Delta \Sigma}$ correlates tightly with the observed aperture mass. The best fit virial mass lies in between the median and the mean actual virial mass when scatter between $V_{\max }$ and luminosity is used.

- We compare the cross bias, $b_{x} \equiv b / r$, measured in simulations and observations and find a good agreement at all probed scales. The correlation coefficient, $r$, obtained for a volume-limited sample similar to that analyzed by S04, is close to unity on scales $\gtrsim 1 h^{-1} \mathrm{Mpc}$. This indicates that the cross bias measured in weak lensing observations does measure the actual bias $b$ of galaxy clustering on these scales.

- We show that for luminous galaxies $\left(M_{r}<-21\right)$ the galaxy-mass correlation function at $r \lesssim 100-$ $200 h^{-1} \mathrm{kpc}$ can be interpreted as the average density profile of these galaxies. We also show that the masses obtained by the $\Delta \Sigma(R)$ measured in weak lensing observations cannot be interpreted in a straightforward way as the mass for galaxies of a given luminosity. In the presence of scatter between mass and light, the obtained mass-luminosity relations should be quoted and interpreted with caution.

We are grateful to Erin Sheldon for invaluable discussions and help understanding the data, for providing his data in electronic format, and for comments on the manuscript. We would also like to thank Anatoly Klypin for running the simulations used in this study, Brandon Allgood for running some of the halo catalogs used, Michael Blanton for communicating to us the results on the scatter between $r$ - and $K$-band luminosities for the SDSS galaxies, Arieh Maller for helpful discussions about scatter and comments on a draft, Chris Miller for allowing us to use results from the Value Added Catalog he has compiled for the SDSS DR1, and Uros Seljak and David Weinberg for useful comments on the manuscript. This work was supported by the National Science Foundation (NSF) under grants No. AST-0206216 and AST-0239759, by NASA through grants NAG5-13274 and NAG5-12326, and by the Kavli Institute for Cosmological Physics at the University 
of Chicago. RHW was supported by NASA through a Hubble Fellowship awarded by the Space Telescope Science Institute, which is operated by the Association of Universities for Research in Astronomy, Inc, for NASA, under contract NAS 5-26555. The simulations used in this study were performed on the IBM RS/6000 SP3 system at the National Energy Research Scientific Computing Center (NERSC) and on the Origin2000 at the National Center for Supercomputing Applications (NCSA). This study has used data from the Sloan Digital Sky Survey (SDSS). Funding for the creation and distribution of the SDSS Archive has been provided by the Alfred P. Sloan Foundation, the Participating Institutions, the National Aeronautics and Space Administration, the National Science Foundation, the U.S. Department of Energy, the Japanese Monbukagakusho, and the Max Planck Society. The SDSS Web site is http://www.sdss.org/.

\section{REFERENCES}

Aaronson, M. \& Mould, J. 1983, ApJ, 265, 1

Abazajian, K. et al. 2003, AJ, 126, 2081

Benson, A. J., Baugh, C. M., Cole, S., Frenk, C. S., \& Lacey, C. G. 2000a, MNRAS, 316, 107

Benson, A. J., Cole, S., Frenk, C. S., Baugh, C. M., \& Lacey, C. G. 2000b, MNRAS, 311, 793

Berlind, A. A. \& Weinberg, D. H. 2002, ApJ, 575, 587

Berlind, A. A., Weinberg, D. H., Benson, A. J., Baugh, C. M., Cole, S., Davé, R., Frenk, C. S., Jenkins, A., Katz, N., \& Lacey, C. G. 2003, ApJ, 593, 1

Blanton, M. R., Brinkmann, J., Csabai, I., Doi, M., Eisenstein, D., Fukugita, M., Gunn, J. E., Hogg, D. W., \& Schlegel, D. J. 2003a, AJ, 125,2348

Blanton, M. R., Eisenstein, D. J., Hogg, D. W., Schlegel, D. J., \& Brinkmann, J. 2003b, astro-ph/0310453

Blanton, M. R. et al. 2003c, ApJ, 592, 819

Brainerd, T. G., Blandford, R. D., \& Smail, I. 1996, ApJ, 466, 623

Bullock, J. S., Kolatt, T. S., Sigad, Y., Somerville, R. S., Kravtsov, A. V., Klypin, A. A., Primack, J. R., \& Dekel, A. 2001, MNRAS, 321,559

Colín, P., Klypin, A. A., Kravtsov, A. V., \& Khokhlov, A. M. 1999, ApJ, 523, 32

Colless, M. et al. 2001, MNRAS, 328, 1039

Colombi, S., Bouchet, F. R., \& Schaeffer, R. 1994, A\&A, 281, 301

Courteau, S., MacArthur, L. A., Dekel, A., Bosch, F. v. d., McIntosh, D. H., \& Dale, D. 2003, ArXiv Astrophysics e-prints

Fischer, P., McKay, T. A., Sheldon, E., \& The SDSS Collaboration. 2000, AJ, 120, 1198

Governato, F., Baugh, C. M., Frenk, C. S., Cole, S., Lacey, C. G., Quinn, T., \& Stadel, J. 1998, Nature, 392, 359

Guzik, J. \& Seljak, U. 2001, MNRAS, 321, 439

-. 2002, MNRAS, 335, 311

Hoekstra, H., Yee, H. K. C., \& Gladders, M. D. 2001, ApJ, 558, L11

-. 2004, ApJ in press, astro-ph/0306515

Itoh, M., Suginohara, T., \& Suto, Y. 1992, PASJ, 44, 481

Kaiser, N. 1984, ApJ, 284, L9

Kannappan, S. J., Fabricant, D. G., \& Franx, M. 2002, AJ, 123, 2358

Katz, N., Hernquist, L., \& Weinberg, D. H. 1999, ApJ, 523, 463

Kauffmann, G., Colberg, J. M., Diaferio, A., \& White, S. D. M. 1999a, MNRAS, 303, 188

-. 1999b, MNRAS, 307, 529

Kauffmann, G., Nusser, A., \& Steinmetz, M. 1997, MNRAS, 286, 795

Klypin, A., Gottlöber, S., Kravtsov, A. V., \& Khokhlov, A. M. 1999, ApJ, 516, 530

Kolatt, T. S., Bullock, J. S., Somerville, R. S., Sigad, Y., Jonsson, P., Kravtsov, A. V., Klypin, A. A., Primack, J. R., Faber, S. M., \& Dekel, A. 1999, ApJ, 523, L109

Kravtsov, A. V. 1999, Ph.D. Thesis

Kravtsov, A. V., Berlind, A. A., Wechsler, R. H., Klypin, A. A., Gottloeber, S., Allgood, B., \& Primack, J. R. 2004a, ApJ in press, astro-ph/0308519

Kravtsov, A. V., Gnedin, O. Y., \& Klypin, A. A. 2004b, ApJ in press, astro-ph/0401088

Kravtsov, A. V. \& Klypin, A. A. 1999, ApJ, 520, 437

Kravtsov, A. V., Klypin, A. A., \& Khokhlov, A. M. 1997, ApJS, 111,
McKay, T. A., Sheldon, E. S., Johnston, D., Grebel, E. K., Prada, F., Rix, H., Bahcall, N. A., Brinkmann, J., Csabai, I., Fukugita, M., Lamb, D. Q., \& York, D. G. 2002, ApJ, 571, L85

McKay, T. A. et al. 2001, astro-ph/0108013

Miralda-Escudé, J. 1991, ApJ, 370, 1

Navarro, J., Frenk, C., \& White, S. 1996, ApJ, 462, 563

-. 1997, ApJ, 490, 493

Neyrinck, M. C., Hamilton, A. J. S., \& Gnedin, N. Y. 2003, astro-ph/0302003

Norberg, P. et al. 2001, MNRAS, 328, 64

Pearce, F. R., Jenkins, A., Frenk, C. S., White, S. D. M., Thomas, P. A., Couchman, H. M. P., Peacock, J. A., \& Efstathiou, G. 2001, MNRAS, 326, 649

Pen, U. 1998, ApJ, 504, 601

Seljak, U. 2000, MNRAS, 318, 203

Sheldon, E. S. et al. 2001, ApJ, 554, 881

-. 2004, AJ in press astro-ph/0312036

Smith, D. R., Bernstein, G. M., Fischer, P., \& Jarvis, M. 2001, ApJ, 551,643

Smith, R. E., Peacock, J. A., Jenkins, A., White, S. D. M., Frenk, C. S., Pearce, F. R., Thomas, P. A., Efstathiou, G., \& Couchman, H. M. P. 2003, MNRAS, 341, 1311

Somerville, R. S., Lemson, G., Sigad, Y., Dekel, A., Kauffmann, G., \& White, S. D. M. 2001, MNRAS, 320, 289

Spergel, D. N. et al. 2003, ApJS, 148, 175

Tegmark, M., Strauss, M., Blanton, M., \& collaboration, S. 2004, Phys.Rev. D in press, astro-ph/0310723

Vale, A. \& Ostriker, J. P. 2004, astro-ph/0402500

van den Bosch, F. C., Yang, X., \& Mo, H. J. 2003, MNRAS, 340, 771

Verheijen, M. A. W. 2001, ApJ, 563, 694

Wechsler, R. H. 2004, in Carnegie Observatoris Astrophysics Series, Vol. 3: Clusters of Galaxies: Probes of Cosmological Structure and Galaxy Evolution, (http://www.ociw.edu/ociw/symposia/series/ symposium $3 /$ proceedings.html)

Wechsler, R. H., Evrard, A. E., McKay, T. A., Miller, C., Nichol, R., Annis, J., \& Bingham, D. 2004, in preparation

Wechsler, R. H., Somerville, R. S., Bullock, J. S., Kolatt, T. S., Primack, J. R., Blumenthal, G. R., \& Dekel, A. 2001, ApJ, 554, 85

Weinberg, D. H., Davé, R., Katz, N., \& Hernquist, L. 2004, ApJ, 601,1

White, M., Hernquist, L., \& Springel, V. 2001, ApJ, 550, L129

Willick, J. A., Courteau, S., Faber, S. M., Burstein, D., Dekel, A., \& Strauss, M. A. 1997, ApJS, 109, 333

Wilson, G., Kaiser, N., Luppino, G. A., \& Cowie, L. L. 2001, ApJ, 555,572

Yang, X. H., Mo, H. J., Kauffmann, G., \& Chu, Y. Q. 2003, MNRAS, 339,387

York, D. G. et al. 2000, AJ, 120, 1579

Yoshikawa, K., Taruya, A., Jing, Y. P., \& Suto, Y. 2001, ApJ, 558, 520

Zehavi, I. et al. 2004, ApJ in press, astro-ph/0301280

Zentner, A., Berlind, A., Kravtsov, A. V., \& Bullock, J. S. 2004, in preparation 